\title{
Support Vector Machine based Stress Detection System to manage COVID-19 pandemic related stress from ECG signal
}

\author{
Md Fahim Rizwan, Rayed Farhad, and Mohammad Hasan Imam, Member, IEEE
}

\begin{abstract}
This study represents a detailed investigation of induced stress detection in humans using Support Vector Machine algorithms. Proper detection of stress can prevent many psychological and physiological problems like the occurrence of major depression disorder (MDD), stress-induced cardiac rhythm abnormalities, or arrhythmia. Stress induced due to COVID -19 pandemic can make the situation worse for the cardiac patients and cause different abnormalities in the normal people due to lockdown condition. Therefore, an ECG based technique is proposed in this paper where the ECG can be recorded for the available handheld/portable devices which are now common to many countries where people can take ECG by their own in their houses and get preliminary information about their cardiac health. From ECG, we can derive RR interval, QT interval, and EDR (ECG derived Respiration) for developing the model for stress detection also. To validate the proposed model, an open-access database named "drivedb" available at Physionet (physionet.org) was used as the training dataset. After verifying several SVM models by changing the ECG length, features, and SVM Kernel type, the results showed an acceptable level of accuracy for Fine Gaussian SVM (i.e. $98.3 \%$ for 1 min ECG and 93.6 \% for 5 min long ECG) with Gaussian Kernel while using all available features (RR, QT, and EDR). This finding emphasizes the importance of including ventricular polarization and respiratory information in stress detection and the possibility of stress detection from short length data (i.e. from 1 min ECG data), which will be very useful to detect stress through portable ECG devices in locked down condition to analyze mental health condition without visiting the specialist doctor at hospital. This technique also alarms the cardiac patients from being stressed too much which might cause severe arrhythmogenesis.
\end{abstract}

Index Terms - Stress, Covid-19 pandemic, SVM, ECG, EDR, RR interval, QT interval, CVD

Md Fahim Rizwan is a student of Masters in Electrical and Electronic Engineering (MEEE), American International UniversityBangladesh, 408/1, Kuratoli, Khilkhet, Dhaka 1229, Bangladesh

Email: fahimrizwan7@gmail.com

Rayed Farhad is a student of Masters in Electrical and Electronic Engineering (MEEE), American International University-Bangladesh, 408/1, Kuratoli, Khilkhet, Dhaka 1229, Bangladesh

Email: rayedfarhad007@gmail.com

Mohammad Hasan Imam is a Senior Assistant Professor of Department of Electrical and Electronic Engineering, American International UniversityBangladesh, 408/1, Kuratoli, Khilkhet, Dhaka 1229, Bangladesh

Email: hasan.imam@aiub.edu

\section{INTRODUCTION}

$\mathrm{S}_{\mathrm{psy}}^{\mathrm{T}}$ TRESS can be commonly interpreted as the disruption in psychological equilibrium. At the point when an individual cannot adjust between the requests that are put on him/her and his/her capacity to adapt to them, at that point, it very well may be called mental pressure or stress. Mental health disturbances or the appearance of the different disease results from the long endurance of stress [1]. The most alarming this is long term stress can initiate several cardiovascular diseases (CVD). The autonomic homeostasis is adversely affected by physiological stress [2]. Ventricular tachyarrhythmias can be triggered by changes in autonomic homeostasis [3]. Proarrhythmic repolarization instability can be increased by increased sympathetic nervous activity leading to spontaneous ventricular arrhythmias. The sympathetic nervous system can be stimulated by physiological stress and this can result in arrhythmogenesis [4]. Stress can induce Catecholaminergic ventricular tachycardia in children with structurally normal heart [5]. Stress can lead reduced heart rate variability, increased QT dispersion, and reduced baroreceptor sensitivity. People with these changes have the utmost risk for emerging fatal ventricular arrhythmias [6,7]. There are two branches of the Autonomic Nervous System (ANS): Sympathetic Nervous System (SNS) and Parasympathetic Nervous System (PNS). During normal condition, the systems are balanced which shows the normal value of heart rate, blood pressure, skin conductance, and muscle activities. During stressed situation, the activation of SNS increases, or that of PNS decreases causing imbalance between two branches which causes arrhythmogenesis [8,9]. Stress can lead to major depressive disorder (MDD). MDD was related to an elevated danger for the advancement of CVD in a recent meta-analysis of prospective studies involving initially physically healthy subjects [10]. One meta-analysis disclosed the relation connecting depression to blood pressure reactivity and reduced heart rate (HR) variability [11]. Another recent report involving more than 1,600 adults demonstrated that stressed state of mind was diagnosed with abnormal systolic pulse and HR variability [12]. Therefore, stress detection and management are critical for cardiovascular health.

Besides the negative impact on CVD, thoughts of selfdestruction are often associated with the involvement of depression, anxiety, and stress with a low degree of life satisfaction. Anyone having thoughts, urges or intentions of committing suicide is called to have suicidal ideation. Many 
factors can increase the risk of suicidal ideation such as family conflicts, increasing competitiveness, lesser resources to deal with the academic stressors, financial issues, exams stress, loneliness, poor academic achievement, and poor physical health. In a research among 9000 students of a Korean High School, it was found that depression due to heavily stressed lifestyle is the strongest predictor of suicidal tendencies [13]. It was also noticed that among those students who have a high depression level are 5.19 times more reluctant to report the thought of suicide and 3.19 times more prone to commit suicide compared to the students who have low depression levels. Depression and anxiety are physiological states, which includes sentiments of vulnerability, misery, absence of delight and enthusiasm for ordinary happenings, and it might occasionally include self-destructive ideations. They are also found to be directly related to the occurrences of high heartbeat, hypertension and several cardiovascular disorders [14]. Stress also reduced the workplace efficiency of the workers and ultimately affect the outcome of the business.

Furthermore, the world is passing the phase of coronavirus pandemic (COVID-19 Pandemic) since December 2019, which is incredibly stressful. Our day to day lifestyles is changing rapidly, and they may never be the same as they used to be. It is not easy for every people to come up with those abrupt changes, which adds additional stress to daily life. This kind of anxiety may include fear of getting affected by the virus or loved ones getting affected, fear of death, loss of job due to lockdown, running out of food, uncertainties in education system, loss of nearest relatives/family members for corona virus attack, lack of proper medical facility to save patients etc. In a recent research about the psychological responses among the general population in China, shows that majority of the respondents have noticeable symptoms of psychological breakdown, severe anxiety, and stress caused by the COVID-19 pandemic situation [35]. In a questionnairebased research, participants showed more negative psychological and emotional responses in response to COVID19 [36]. Healthy physical activity generated from stable immunological development which is a necessary factor to fight with contagious attacks like Corona virus is hampered directly by psychological shocks or events of severe stress. Moreover, the incidence of suicide where stress plays a key role for developing this self-termination tendency around the world including Bangladesh increases after the pandemic starts [37]. The international committee of Red Cross declared that pandemic situation due to COVID-19 may cause to increase psychological stress exponentially within human around the world because of increased depression and anxiety for the loss of near ones, fear of death from infection and huge uncertainties about future life. Doctors at the National Institute of Mental Health and Hospital, Bangladesh concluded from the last three to four months experience that many people in Bangladesh also are suffering from depression for a long time and may suffer post-traumatic disorder (PTSD) which can cause other physical abnormalities due to Covid-19 situation. A recent study on Bangladeshi adult population indicates that about $85.60 \%$ of the participants are suffering from COVID19-related stress attack, which results in sleep disorder, unstable psychological behavior, and deterioration in everyday life-style [37]. Many patients are affected with cardiovascular abnormalities due to heavy stress and anxiety. However, during the lockdown condition it is difficult to have proper consultancy with a cardiologist. Therefore, these patients will be benefitted if they have stress detection system which will prevent to make situation worse. As a result, detection of stress through easily available techniques is crucial for betterment of lifestyle and development of society.

Stress can normally be detected from various Biosignal and questionnaire method used in psychiatric techniques. Galvanic skin response (GSR) was found to drop during stressed situation [15]. Using electro dermal activity (EDA) detectors, skin electrical conductivity can be measured. Changes in EDA can trigger the sympathetic nervous system (SNS) activity which happens when a person is stressed, which makes EDA a useful signal to detect stress [16]. Research also showed that the muscle activity recorded by EMG is altered during stressed situation, which can be used to detect stress. However, the main challenge in EMG signal processing is the removal of noise and feature extraction. Changes in skin temperature can also help us measuring stress level too [17-20]. Detection of stress from ECG were done by heart rate (i.e. RR interval from ECG) and heart rate variability (HRV) analysis [21]. A Questionnaire method was used to assess stress at workplace where 4800 female employees took part in a 98 questionnaire items [22]. EMG, respiration, skin conductivity and heart rate were analyzed in 24 subjects during driving to measure real time stress using machine learning technique [23]. A research on combined physical and mental workload-based stress measurement showed that high frequency component of HRV and blood pressure variability changes remarkably [24]. Ventricular repolarization (VR) characteristics represented by QT interval whose abnormality is the main cause of sudden cardiac death in ECG are also affected by stress [25-27]. People having stressed condition may be identified by the prolongation of QT interval due to abnormal activation of SNS. QT intervals are shortened in some studies when a person is stressed [28]. Respiration and cardiovascular system abnormality are strongly interconnected with SNS activation during stress [29]. Stressed individual has a faster and shallower breathing pattern [30]. Respiratory rate and respiration amplitude are changing with SNS activity, which directs to stress [29]. In 2015, Nermine Munla et al. explored driver stress using ECG signal feature (only RR interval-based features) using machine learning. The study detect stress with an accuracy of $83 \%$ [31]. In 2016, Ya Jun Yu et al. analyzed the effects of using GPS navigation on drivers. Using high and low density of GPS instructions, the study showed that RR intervals, SDRR, and HRV are the most efficient parameters that indicate stress [32]. Ahmad Rauf Subhani et al. developed a machine-learning framework using EEG signal analysis of stressed subjects, which produced $94.6 \%$ accuracy for 2 - level identification and $83.4 \%$ accuracy in the case of multiple level identification [33]. However, EEG signal acquisition and processing are complex in comparison of ECG signal analysis for analyzing mental state transition. Moreover, respiratory system recoding requires devices which might be not suitable 
for long term continuous use. Therefore, the main objective of this study is to propose a technique using machine learning techniques for the detection of stress from ECG which is easily recordable now through the use of currently available wearable and portable devices. Another motivation of using ECG is that HRV, VR characteristics and respiratory information can be extracted from ECG only as reported in $[25,34]$. As a result, the proposed system can only need to record and analyze ECG in contrast to multi signal processing and analysis. Until now, almost all other methods have used only RR interval from ECG as the main signal to detect stress. In our research, we used QT interval, RR interval and EDR (ECG Derived Respiration) combinedly to detect stress. We have chosen EDR (ECG Derived Respiration) also extracted from ECG only as an alternate for respiration signal as they show similar properties and be used as successfully in some previous studies [25]. Consequently, no external sensors were needed to record respiration signal. Another important motivation of this research is to investigate the length of ECG (i.e. whether short or long ECG recording is needed) to detect stress with high accuracy. To the best of our knowledge, no previous study has used respiratory information from ECG (ECG Derived Respiration or EDR) and QT interval in stress detection using machine learning techniques. The proposed designed system of this research may be helpful to detect stress easily and to take necessary actions during this COVID -19 pandemic. The initial findings of this study have already presented in [34].

\section{RESEARCH METHOD AND MODELLING}

This section describes steps followed for developing the model and the ECG data processing. ECG data were collected from an open access database in Physionet's "drivedb" [23] database which contain some Biosignal recordings along with ECG to monitor the stress level changes in healthy adult drivers when driving conditions were changed. Supervised machine learning method was chosen for modelling analysis in MATLAB 9.0 R2016a [38]. A Graphical User Interface (GUI) was designed using MATLAB's APP DESIGNER, where if the user loads an ECG file of any patient, the system will tell if the patient is stressed or not.

\section{A. ECG data collection and Feature Extraction}

For validation of the model, Stress Recognition in Automobile Drivers database ("drivedb") available at Physionet (www.Physionet.org) was utilized where some healthy subjects' normal and stressed condition's ECG information were recorded [23]. In this database, an exploratory convention was planned for the identification of stress level changes due to driving in different road traffic condition which causes changes in different physiological signals of the healthy drivers. As per the designed system, subjects were driving a vehicle following a set course and their physiologic responses were observed by investigating a few recorded physiological signals like Electrocardiogram (ECG), Electromyogram (EMG), skin conductivity and respiration [23]. The driving convention was intended to take the driver in various street conditions with variable traffic to such an extent that various degrees of stress were probably likely to be induced. A total number of 15 healthy subject's information was taken in this study for model validation. In our examination, we have used 5 minutes of ECG signal during resting condition and high-stress conditions when the drivers were involved in driving in heavy traffic and complex road patterns. The drivers ' resting conditions were assumed to be "Not Stressed" and driving in heavy traffic situations were considered to be "Stressed."

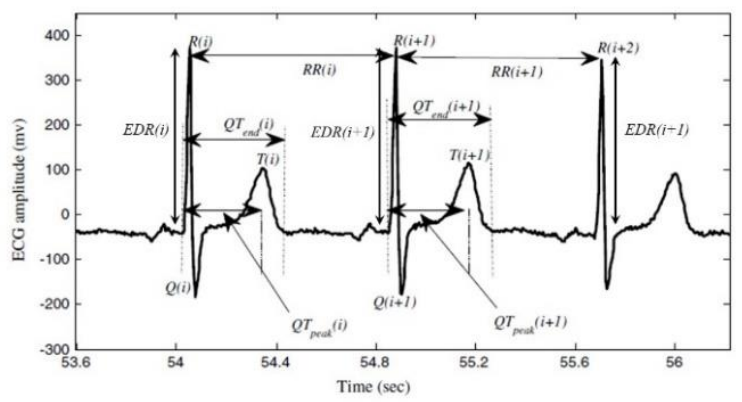

Figure 1. Various ECG wave parameters (Q, R, and T waves) and RR and QT duration calculation (QTpeak and QTend) for three heartbeats [25].

First a median filter was applied to reduce the baseline wandering noise. ECG signal was filtered for powerline interference and recorded in the database. ECG signals were then processed for features extraction. RR interval and QT interval was detected by detecting the $\mathrm{R}$ peak, $\mathrm{Q}$ peak, $\mathrm{T}$ wave peak and T wave endpoints by using Berger's algorithm. EDR signal was derived as the variation of $R$ wave amplitude. The whole procedure of feature extraction was described in an earlier article [25,34] and the extracted features are shown in figure 1. For modelling purpose, we used QTend interval as QT interval the Tpeak to Tend interval is found to contain important information about autonomic nervous system activation due to stress induction.

Figure 2 shows the how the ECG data and decision variables are processed for Model Training. After choosing the data, they were imported to the classification learner.

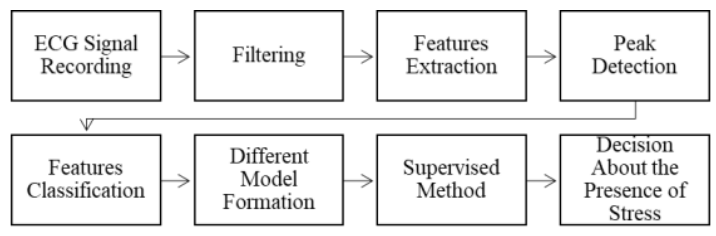

Figure 2: Block Diagram of the proposed Stress Detection System.

\section{B. Modelling and Validation Techniques}

The modelling and validation were done according to the block diagram in figure 3. Classification Learner app from MATLAB's Machine Statistics and Machine Learning Toolbox was used to train and validate the model. In this study, two types of model validations were done by using short length and long length ECG signal of each subject. At first, the model was trained with short length ECG segments. 
A total 2500 Second of data (i.e. almost $85 \mathrm{sec}$ of data[1 to 1.5 min long] for each subject of a total of 15 subjects, both stressed and relaxed or not stressed) was used to train the model. Then the model was trained with long length ECG. A total 7350 Second of data (i.e. 4-5 minutes of data for each subject, both stressed and relaxed) was used to train the model. The effect of different features on model performance were analyzed to verify whether increasing the number of features actually increase the detection efficiency and which feature are important to add in the model.

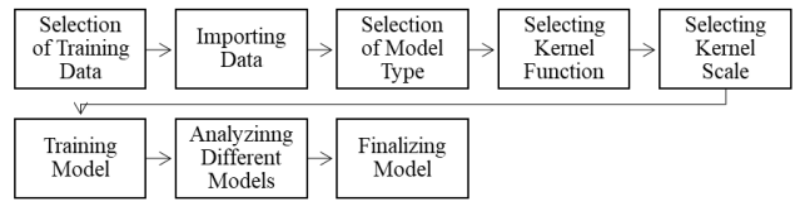

Figure 3: Workflow Diagram of SVM Model analysis in MATLAB

Figure 4 depicts the pattern of training data which was given as model input. Supervised Machine Learning was used to detect stress in this research. The ECG features chosen for stress monitoring are QT interval, RR interval and ECG Derived Respiration (EDR). The system will determine whether the subject is stressed or relaxed by analyzing the dynamics between these ECG features through modelling. Because the data have two category tags (i.e. stressed and not stressed or relaxed), Support Vector Machine (SVM) a supervised machine learning technique was chosen to classify and detect stress.

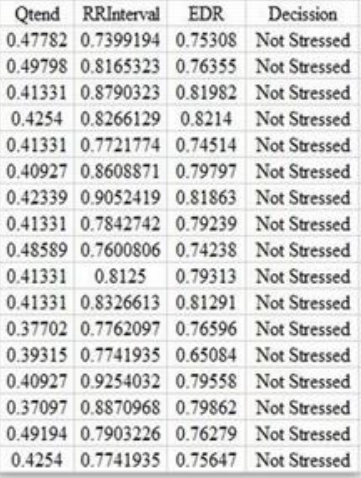

\begin{tabular}{|c|c|c|c|}
\hline Qtend & RRInterval & EDR & Decission \\
\hline 0.3629 & 0.9012097 & 1.48158 & Stressed \\
\hline 0.36492 & 0.9193548 & 1.54413 & Stressed \\
\hline 0.36694 & 0.8245968 & 1.45752 & Stressed \\
\hline 0.35484 & 0.8830645 & 1.47348 & Stressed \\
\hline 0.36492 & 0.9637097 & 1.50651 & Stressed \\
\hline 0.37097 & 0.8447581 & 1.37285 & Stressed \\
\hline 0.3629 & 0.8870968 & 1.48037 & Stressed \\
\hline 0.36492 & 0.8991935 & 1.49324 & Stressed \\
\hline 0.3629 & 0.8165323 & 1.47152 & Stressed \\
\hline 0.36492 & 0.9012097 & 1.50668 & Stressed \\
\hline 0.35887 & 0.9495968 & 1.54069 & Stressed \\
\hline 0.34476 & 0.8447581 & 1.47174 & Stressed \\
\hline 0.35685 & 0.9112903 & 1.48052 & Stressed \\
\hline 0.36694 & 0.9798387 & 1.53021 & Stressed \\
\hline 0.36895 & 0.9012097 & 1.51735 & Stressed \\
\hline 0.36694 & 0.8830645 & 1.45438 & Stressed \\
\hline 0.36492 & 0.9334677 & 1.50834 & Stressed \\
\hline
\end{tabular}

(b)
Figure 4: Snapshot of the extracted features for ECG that are used in training the model.

Although support Vector Machine (SVM) is suitable for discrete or continuous data sets, it is primarily used for discrete sets of data classification technique. In this process, the data are shown in an $\mathrm{n}$-dimensional plane where $\mathrm{n}$ is the number of features. Classification is then carried out by locating the hyperplane that differentiates between the two groups. The model was trained after selecting the validation method, model type, and Kernel Functions. Models can be finalized after analyzing the accuracy, ROC curve and confusion matrices of those models. After finalizing the model, an app was designed with MATLAB App Designer which can be used to detect stress in real-time.

\section{RESULT ANALYSIS}

Initially, the model was trained using all the three ECG based features with the basic SVM model types (Linear, Quadratic, Cubic, and Gaussian) utilizing the default Kernel Functions in the MATLAB Classification Learner App version 2016a. We analyzed both short length ECG data (i.e. around 1-minute long ECG data) and long length ECG data (i.e. 5 minutes data) to validate different model performance in detecting stress. The validation plan was selected as $\mathrm{k}$ fold cross-validation, which is usually the preferred method for large datasets, because it gives the model the opportunity to train on multiple train-test splits. We used 10-fold cross validation scheme for best model selection. This gives a better indication of how well the model will perform on unseen data. In $\mathrm{k}$-fold cross validation, the data is divided into $\mathrm{k}$ number of sets. Among them, one set is used as test data set and other sets are used as training data set. This process is repeated $\mathrm{k}$ times where every single set gets a chance to be the test set once. This feature allows k-fold cross validation to eliminate model overfitting [34]. All three features, QT interval, RR interval, and ECG Derived Respiration (EDR) were used for analyzing for both short and long length ECG data. The best model can be selected by evaluating the model accuracy values which is calculated from the sensitivity and specificity values and the distribution of confusion matrix and ROC curves.

Table 1 displays the model accuracy of various forms of SVM using all the features were evaluated for model analysis and Gaussian SVM showed acceptable results for both the short and long length ECG data.

To investigate the impact of various ECG features on stress, the model was then trained utilizing two features to check whether it is feasible to detect stress. A significant drop in the model accuracy was found if we use combination of two feature as shown in Table 2 for both short and long ECG segments. Combination of QT interval and RR interval was found to give an accuracy of almost $95 \%$ for cubic SVM model in ECG length of 1 minute. Fine Gaussian models generated over $90 \%$ accuracy for determining stress using QT and EDR for both short- and long-term ECG signal. Other models failed to generate comparable amount of accuracy using combination of two features (Table 2).

Almost all of the model-based stress detection techniques found in the literature have used only one feature, mostly RR interval to detect stress as found in the literature $[6,8,25]$. But their performance is not acceptable for biomedical applications (i.e. average Accuracy level less than 90\%). Therefore, in this study we trained the model with QT interval, RR interval and ECG Derived Respiration (EDR) separately and analyzed the results. We also analyzed the model performance for detecting stress using single feature (Table 3 ). It was found that in case of both short and long length ECG, any single feature of ECG cannot detect the stress related changes in ECG dynamics.

Table 1, 2 and 3 describes the modelling results by using the default Kernel Function types available for every model type. Kernel Function can likewise be tuned to improve the 
model performance. To achieve this goal, we have trained the models with several types of Kernel Functions. Table 4 showed the results of variation of model accuracy when Kernel Function is varied for short length data. In MATLAB classification learner app, we have four Kernel Functions available for SVM evaluation. Linear and Quadratic Kernel
Functions did not show satisfactory results. Only Gaussian and Cubic Kernel Function types showed promising results in detecting stress using all three features of ECG as shown in Table 4.

Table 1: Model accuracy for different types of SVM model using all the features and the default Kernel Function built-in MATLAB

\begin{tabular}{|c|c|c|c|}
\hline Model Name & Features Used & $\begin{array}{l}\text { Accuracy for Short } \\
\text { length ECG }\end{array}$ & $\begin{array}{l}\text { Accuracy for Long } \\
\text { length ECG }\end{array}$ \\
\hline Linear SVM & \multirow{4}{*}{$\begin{array}{l}\text { QT interval, RR interval, } \\
\text { EDR }\end{array}$} & $52.6 \%$ & $52.5 \%$ \\
\hline Quadratic SVM & & $88.6 \%$ & $49.7 \%$ \\
\hline Cubic SVM & & $97.2 \%$ & $66.2 \%$ \\
\hline Fine Gaussian SVM & & $98.3 \%$ & $93.6 \%$ \\
\hline
\end{tabular}

Table 2: Model accuracy variation by using combination of two features used for modelling

\begin{tabular}{|l|l|l|l|l|l|l|}
\hline Model Name & Accuracy level for Short length ECG data & \multicolumn{3}{|l|}{ Accuracy level for long length ECG data } \\
\hline Features & QT, RR & QT, EDR & RR, EDR & QT, RR & QT, EDR & RR, EDR \\
\hline Linear SVM & $50.6 \%$ & $52.6 \%$ & $61.5 \%$ & $52.2 \%$ & $51.7 \%$ & $55.4 \%$ \\
\hline Quadratic SVM & $83.5 \%$ & $86.3 \%$ & $51.2 \%$ & $53.1 \%$ & $51.7 \%$ & $58.3 \%$ \\
\hline Cubic SVM & $94.9 \%$ & $89.2 \%$ & $53.1 \%$ & $47.7 \%$ & $48.7 \%$ & $59.6 \%$ \\
\hline Fine Gaussian SVM & $84.6 \%$ & $93.2 \%$ & $75.8 \%$ & $83.9 \%$ & $93.2 \%$ \\
\hline
\end{tabular}

Table 3: Model accuracy variation by using only one feature used for modelling

\begin{tabular}{|l|l|l|l|l|l|l|}
\hline Model Name & Accuracy level for Short length ECG data & \multicolumn{3}{|l|}{ Accuracy level for long length ECG data } \\
\hline Features & QT & RR & EDR & QT & RR \\
\hline Linear SVM & $50.5 \%$ & $61.3 \%$ & $48.6 \%$ & $50 \%$ & $50.5 \%$ & EDR \\
\hline Quadratic SVM & $30.6 \%$ & $40.8 \%$ & $48.7 \%$ & $50.3 \%$ & $50.9 \%$ & $42 \%$ \\
\hline Cubic SVM & $61.5 \%$ & $54.5 \%$ & $49 \%$ & $46.5 \%$ & $44 \%$ & $54 \%$ \\
\hline Fine Gaussian SVM & $81.7 \%$ & $61.2 \%$ & $63.7 \%$ & $81.1 \%$ & $60.3 \%$ \\
\hline
\end{tabular}

Table 4: Model performance variation by changing Kernel Functions for short length ECG data

\begin{tabular}{|l|l|l|}
\hline Model Type & Kernel Function & Accuracy \\
\hline \multirow{2}{*}{ Linear SVM } & Gaussian & $98.6 \%$ \\
\cline { 2 - 3 } & Cubic & $97.2 \%$ \\
\hline \multirow{2}{*}{ Quadratic SVM } & Gaussian & $98.6 \%$ \\
\cline { 2 - 3 } & Cubic & $97.1 \%$ \\
\hline \multirow{2}{*}{ Cubic SVM } & Gaussian & $98.6 \%$ \\
\cline { 2 - 3 } & Cubic & $97.2 \%$ \\
\hline
\end{tabular}

To validate whether short length or long length ECG data was useful for detecting stress, we further increased the number of ECG feature samples from 2500 bits to 7350 bits (i.e. $1 \mathrm{~min}$ to $5 \mathrm{~min}$ ). As discussed earlier, the model performs better if we consider three features of ECG signal (i.e. QT interval, RR interval and ECG Derived Respiration), so for cross validation, we have used those three features of long length ECG signal which contain almost 5 min of data. The model accuracy values with $\mathrm{k}=10$, which is commonly used cross validation scheme are shown in table 5. Manual kernel scale was only can be varied for the different Gaussian type SVMs and the optimized values are shown in the following tables.

Table 5: Model accuracy by varying the Kernel Function for long length ECG data

\begin{tabular}{|l|l|l|}
\hline Name & Kernel Function & Model Accuracy $(\%)$ \\
\hline \multirow{4}{*}{ Linear SVM } & Gaussian & 92.3 \\
\cline { 2 - 3 } & Linear & 53.5 \\
\cline { 2 - 3 } & Cubic & 53.6 \\
\cline { 2 - 3 } & Quadratic & 55.2 \\
\hline \multirow{3}{*}{ Quadratic SVM } & Gaussian & 91.8 \\
\cline { 2 - 3 } & Linear & 53.5 \\
\cline { 2 - 3 } & Cubic & 52.6 \\
\hline
\end{tabular}




\begin{tabular}{|l|l|l|}
\hline & Quadratic & 53.8 \\
\hline \multirow{2}{*}{ Cubic SVM } & Gaussian & 92.3 \\
\cline { 2 - 3 } & Linear & 53.5 \\
\hline
\end{tabular}

From table 5, it can be noticed that Gaussian Kernel Function of different SVM models shows better accuracy than other kernels in all of the considered SVM types as found for short length of ECG data.

From the above analysis, we found that Gaussian Kernel Function gave better accuracy for different types of SVM models and Fine Gaussian SVM showed most reliable performance in terms of detecting stress for both short- and long-term ECG segment analysis.

A Graphical User Interface (GUI) was designed using MATLAB's APP DESIGNER. This interface will ask the user to select the ECG file (.m or. mat format only) from computer's memory. After selecting the ECG, user has to enter the sampling frequency of the corresponding ECG and enter a filename. If all the fields are correctly filled, it will show "LOADED" after clicking the LOAD button. Then the stored program detects the RR, QT and EDR from the ECG and the filename is the name of the excel file that will be created by the program to store QT, RR and EDR data of the corresponding ECG. After sometimes, the program will classify the ECG and show decision. Our designed GUI looks like figure 5 .

Figure 6 represents the results of the GUI. Figure 6(a) represents the view if the selected person's ECG is stressed. On the other hand, if the selected ECG is not stressed, the GUI will look like Figure 6(b).

\section{DISCUSSION}

In this study, the focus was on detecting human stress using distinctive features extracted from ECG signal only through machine learning techniques. For detecting stress, supervised machine learning was used as both input data types and possible detection CLASS types were known. Among different supervised machine learning methods, support vector machine (SVM) was used as this a supervised machine learning problem and SVM showed reliable performance in several studies in this type of biosignal based classification problems with long data [30-33] so that our results can be comparable with other results.

We investigated the effect of ECG data length, different number of features and the effect of Kernel Function variation on model performance. The detection or classification performance is mainly analyzed from the Accuracy feature of model simulation output. The value of accuracy is calculated from the Confusion matrix parameters (i.e. TP, TN, FP, FN). Variation of model accuracies were observed by changing Kernel Functions of different SVM models for both short and long length ECG data. From Table 1, it was found that the model can only detect stress from both short and long length ECG data if Fine gaussian SVM was used. Liner and Quadratic SVMs cannot be utilized for the detection of stress as it demonstrates less and unacceptable level of accuracy.
From the classification learner app, the confusion matrix of linear SVM was examined and it was noticed that the amount of correctly detected stressed and relaxed information is just $44 \%$ and $61 \%$ respectively. Cubic SVM gave an accuracy of 97.2\% with default Kernel Function (i.e. Cubic Kernel type) only for short length ECG data but not for long length ECG. However, if the Kernel Function was changed for the chosen model, the accuracy level increased significantly as found in Table 4 for short length ECG data. If Gaussian Kernel Function was used for Linear, Quadratic and Cubic SVM model analysis, the accuracy level was found to be over $95 \%$ in all cases. Figure 7 shows the confusion matrix for the Cubic SVM after changing the default Kernel Function. It was found that quantity of accurately detected stressed and relaxed information of $98 \%$ and $97 \%$ separately. Therefore, it can be concluded that Kernel Function has an effect on model performance and for our study it was found that Gaussian Kernel Function performed better than any other kernel type for detecting stress from ECG derived feature. We also investigated which features derived from ECG were critical for detecting stress level. If two features were used to train the model, the model accuracies were also found to be low in comparison to that model performance using three features. Table 1 represents the results for both $1 \mathrm{~min}$ and $5 \mathrm{~min}$ long ECG data segments using all three features. For models using two features, Fine Gaussian SVM showed some acceptable level of performance for detection using QT and EDR interval for both short- and long-term ECG. Cubic SVM can also detect stress successfully for QT and RR based models for short length ECG data. Therefore, it can be concluded that the model will be more accurate if we use more relevant features from ECG signal. Moreover, if QT interval and Respiratory information (EDR) are used as one of the two features, the model performs better than the models without QT interval. Therefore, it can be said that QT interval and EDR are important parameters to detect stress as established by some recent studies [25, 30,31,34]. This modelling study also supports the findings of relation of stress with ventricular polarization (i.e. QT interval) and respiration phenomena (i.e. EDR). The Models cannot detect stress if only one feature was used as shown in Table 3. This validates the dynamical relationship between heart rate, ventricular polarization and respiratory information due to stress induction and stress level changes. In conclusion, it can be said that all the features derived from ECG used in this study should be used for accurate model design for the detection of stress and analysis of stress level transition.

This study also analyzed whether short length ECG (e.g. 1 min long) and ECG segment of length of 5 min was better for detecting stress using modelling. For short term data, Fine Gaussian SVM performs better that other SVM types for the detection of stress. However, as the number of data is increased, model accuracy value is decreasing than the short- 
term data as shown in table 1. Therefore, it can be concluded that Fine Gaussian SVM with Gaussian Kernel Function was the best choice for both Short- and long-term ECG based Stress detection technique and short-term ECG data (i.e. 1 min long ECG) can be used for reliable detecting stress.

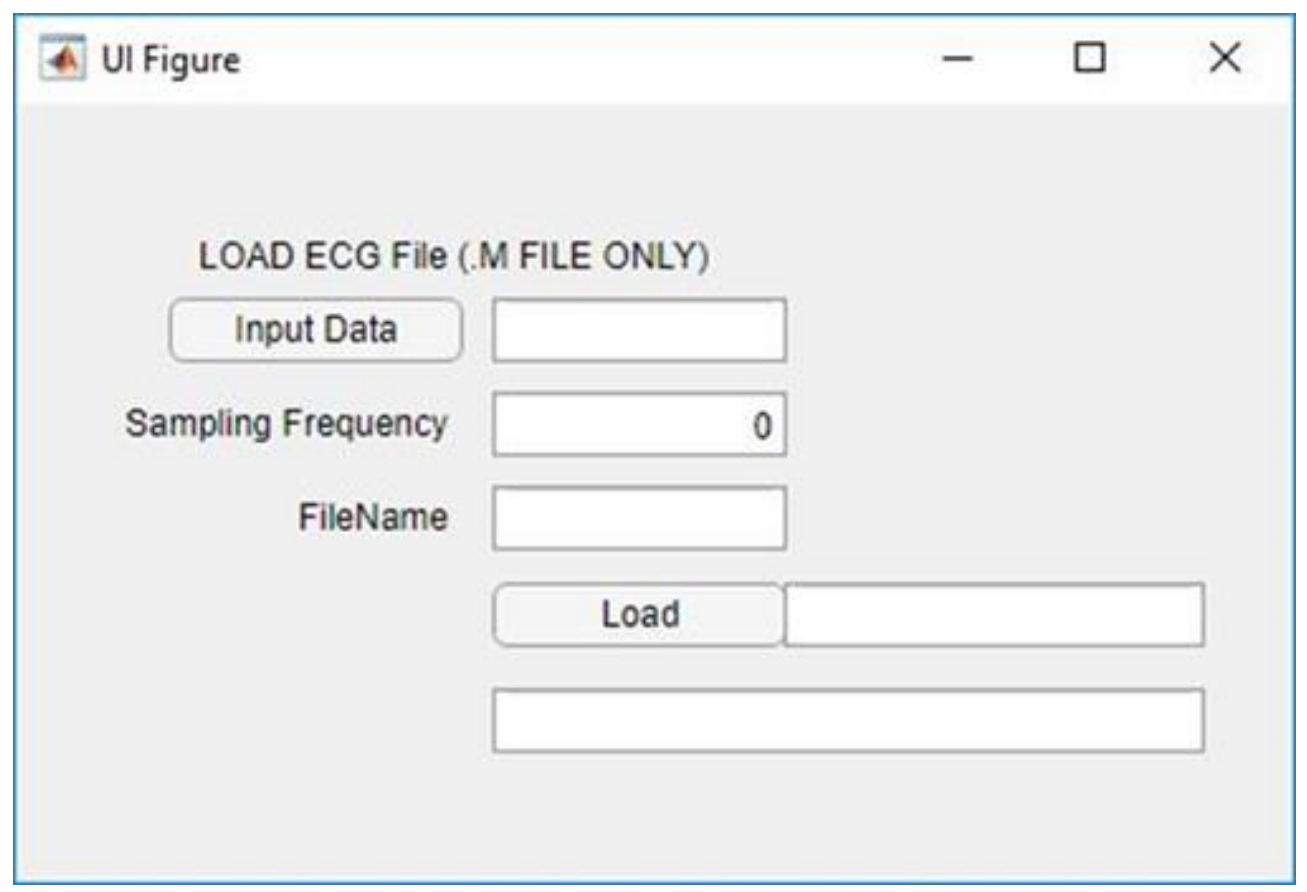

Figure 5: Graphical User Interface (GUI) to detect stress.

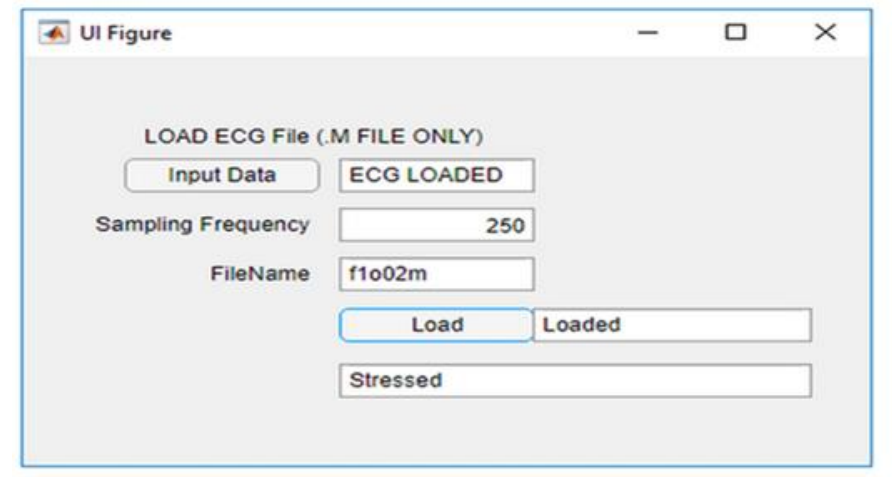

(a)

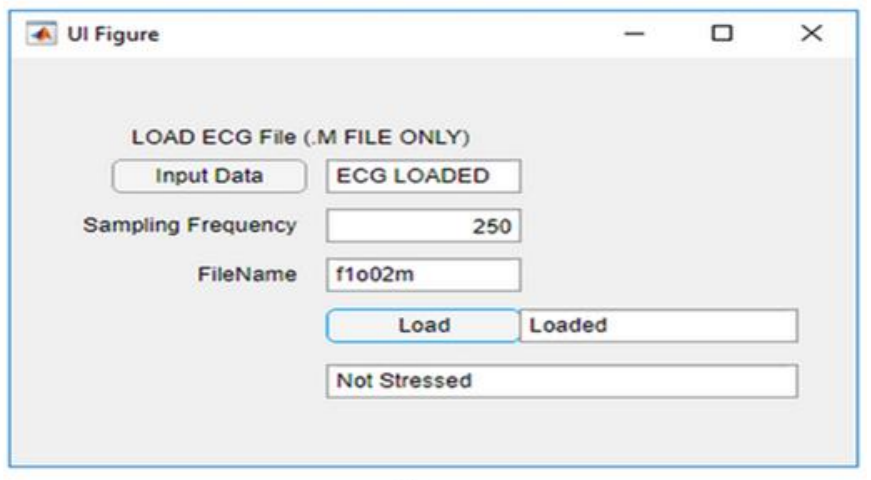

(b)

Figure 6: Results of GUI under different conditions. (a) If the subject is stressed, (b) If the subject is not stressed
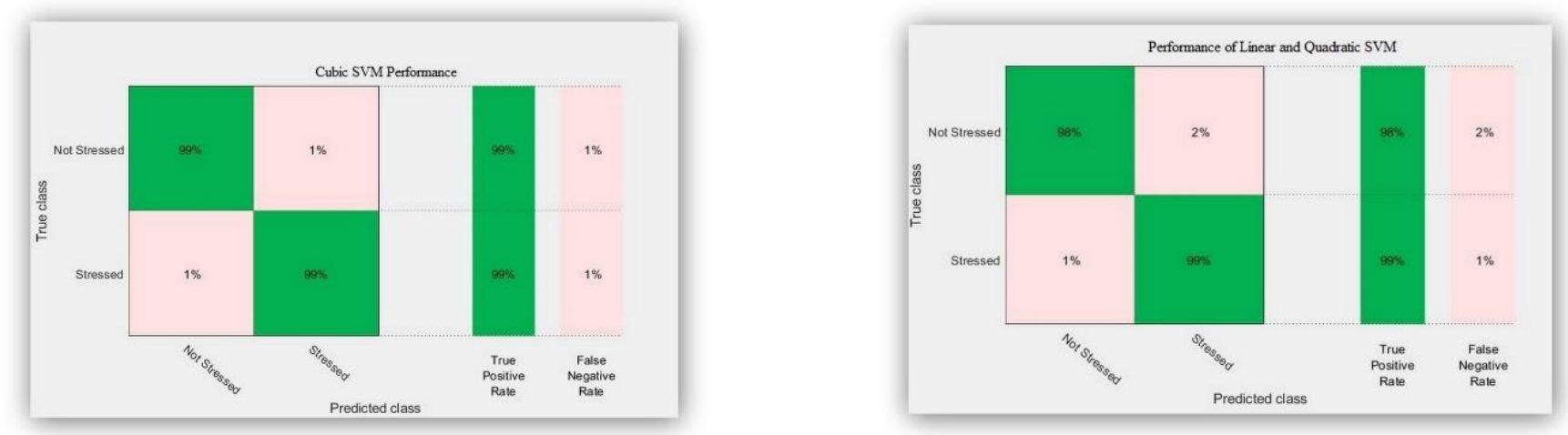

Figure 7: Confusion Matrix for the Linear, Quadratic and Cubic SVM using Gaussian Kernel Function. 


\section{LIMITATIONS}

This study proposed a machine learning based model from ECG signal features for stress detection. However, ECG recording techniques and feature analysis techniques might not always be available for low income group of people and all rural medical facilities. The wearable ECG recording devices also is not much popular in the middle to low income countries. Therefore, awareness of this technology and benefit of having opportunities to analyze critical medical information from this wearable /handheld portable biosignal recording devices should be advertised both from the government level and personal level. Future work will be focused on how efficiently the ECG data from the wearable recorders can be analyzed using the proposed system.

\section{CONCLUSION}

In this paper, different supervised machine learning models to detect stress have been analyzed using multiple ECG features such as QT interval, RR interval, and EDR. This method to detect stress from ECG signal can help an individual to assess one's psychological condition as well as physical condition, from which he/she will be able to take necessary precautions. It was found that ECG derived respiration (EDR) and QT interval are important parameters to detect stress. It can also be concluded that, short term ECG signals can be used for effective detection of stress which is a particularly important finding. That indicates that the model demonstrated in this study can be compatible with portable ECG devices in detecting stress as they allow short term ECG recording. Application of the proposed technique can help the people under lockdown or quarantine condition during COVID-19 pandemic to detect the mental stress which assist to improve their lifestyle and guide them to decide whether it is necessary to have a visit to a psychiatrists for advice and counselling. The technique helps the cardiac patients by alarming the caregiver or the person by detecting if they are stressed or not as stress will deteriorate the condition of cardiac health.

\section{ACKNOWLEDGMENT}

This research was supported by the Department of Electrical and Electronic Engineering, Faculty of Engineering, American International University-Bangladesh (AIUB), Dhaka-1229, Bangladesh.

\section{REFERENCES}

[1] Kalisiewicz, D. "A Small Encyclopedia of PWN." (2000). Scientific Publishers PWN; Warsaw, Poland: 2000. Stress; p. 778.

[2] Das, Sajal, and James H. O'Keefe. "Behavioral cardiology: recognizing and addressing the profound impact of psychosocial stress on cardiovascular health." Current atherosclerosis reports 8, no. 2 (2006): 111-118.

[3] Shusterman, Vladimir, Benhur Aysin, Venkateshwar Gottipaty, Raul Weiss, Susan Brode, David Schwartzman, and Kelley P. Anderson. "Autonomic nervous system activity and the spontaneous initiation of ventricular tachycardia." Journal of the American College of Cardiology 32, no. 7 (1998): 1891-1899.

[4] Brodsky, Michael A., David A. Sato, Lloyd T. Iseri, Larry J. Wolff, and Byron J. Allen. "Ventricular tachyarrhythmia associated with psychological stress: the role of the sympathetic nervous system." JAMA 257, no. 15 (1987): 2064-2067.

[5] Leenhardt, Antoine, Vincent Lucet, Isabelle Denjoy, Francis Grau, Dien Do Ngoc, and Philippe Coumel. "Catecholaminergic polymorphic ventricular tachycardia in children: a 7-year follow-up of 21 patients." Circulation 91, no. 5 (1995): 1512-1519.

[6] Johnson, Beena, and Johnson Francis. "Stress and cardiac arrhythmias." Indian pacing and electrophysiology journal 14, no. 5 (2014): 230.

[7] Brunckhorst, C. B., J. Holzmeister, C. Scharf, C. Binggeli, and F. Duru. "Stress, Depression und kardiale Arrhythmien." Therapeutische Umschau 60, no. 11 (2003): 673-681.

[8] Taggart, Peter, and Pier Lambiase. "Anger, emotion, and arrhythmias: from brain to heart." Frontiers in physiology 2 (2011): 67.

[9] Andreassi George, L. "Psychophysiology: human behavior and physiological response (Psychology of the press).

[10] Rugulies, Reiner. "Depression as a predictor for coronary heart disease: a review and meta-analysis." American journal of preventive medicine 23, no. 1 (2002): 51-61.

[11] Kibler, Jeffrey L., and Mindy Ma. "Depressive symptoms and cardiovascular reactivity to laboratory behavioral stress." International Journal of Behavioral Medicine 11, no. 2 (2004): 81-87.

[12] Carroll, Douglas, Anna C. Phillips, Kate Hunt, and Geoff Der. "Symptoms of depression and cardiovascular reactions to acute psychological stress: evidence from a population study." Biological psychology 75, no. 1 (2007): 68-74.

[13] Juon, Hee-Soon, Jung Ja Nam, and Margaret E. Ensminger. child psychology and psychiatry 35, no. 4 (1994): 663-676.

[14] Naseem, Sabahat, and Seema Munaf. "Suicidal ideation, depression, anxiety, stress, and life satisfaction of medical, engineering, and social anxiety, stress, and life satisfaction of medical, engineering, and social (2017): 422-427.

[15] Shi, Yu, Natalie Ruiz, Ronnie Taib, Eric Choi, and Fang Chen. "Galvanic skin response (GSR) as an index of cognitive load." In CHI'07 extended abstracts on Human factors in computing systems, pp. 2651-2656. 2007.

[16] Lundberg, Ulf, Roland Kadefors, Bo Melin, Gunnar Palmerud, Peter Hassmén, Margareta Engström, and Ingela Elfsberg Dohns. International journal of behavioral medicine 1, no. 4 (1994): 354-370.

17] Wijsman, Jacqueline, Bernard Grundlehner, Julien Penders, and Hermie Hermens. "Trapezius muscle EMG as predictor of mental stress." In Wireless Health 2010, pp. 155-163. 2010.

[18] Crescentini, Cristiano, Luca Chittaro, Viviana Capurso, Riccardo Sioni, and Franco Fabbro. "Psychological and physiological responses to stressful situations in immersive virtual reality: Differences between users who practice mindfulness meditation and controls." Computers in Human Behavior 59 (2016): 304-316.

[19] Yamakoshi, Takehiro, K. Yamakoshi, S. Tanaka, M. Nogawa, SangBum Park, Mariko Shibata, Y. Sawada, P. Rolfe, and Yasuo Hirose. "Feasibility study on driver's stress detection from differential skin temperature measurement." In 2008 30th Annual International Conference of the lEEE Engineering in Medicine and Biology Society, pp. 1076-1079. IEEE, 2008.

[20] Taelman, Joachim, Steven Vandeput, Arthur Spaepen, and Sabine Van Huffel. "Influence of mental stress on heart rate and heart rate variability." In 4th European conference of the international federation for medical and biological engineering, pp. 1366-1369. Springer, Berlin, Heidelberg, 2009.

[21] Hladký, A. "A questionnaire technique for assessing the stress at work." Journal of hygiene, epidemiology, microbiology, and immunology 28 , no. 4 (1984): 383-398.

[22] Boucsein, Wolfram. Electrodermal activity. Springer Science \& Business Media, 2012.

[23] Healey, Jennifer A., and Rosalind W. Picard. "Detecting stress during real-world driving tasks using physiological sensors." IEEE Transactions on intelligent transportation systems 6 , no. 2 (2005): 156-166. 
[24] Hjortskov, Nis, Dag Rissén, Anne Katrine Blangsted, Nils Fallentin, Ulf Lundberg, and Karen Søgaard. "The effect of mental stress on heart rate variability and blood pressure during computer work." European journal of applied physiology 92, no. 1-2 (2004): 84-89.

[25] Imam, Mohammad Hasan, Chandan K. Karmakar, Ahsan H. Khandoker, and Marimuthu Palaniswami. "Effect of ECG-derived respiration (EDR) on modeling ventricular repolarization dynamics in different physiological and psychological conditions." Medical \& biological engineering \& computing 52, no. 10 (2014): 851-860.

[26] Kohavi, Ron. "A study of cross-validation and bootstrap for accuracy estimation and model selection." In Ijcai, vol. 14, no. 2, pp. 1137-1145. 1995.

[27] Karmakar, Chandan, Mohammad Hasan Imam, Ahsan Khandoker, and Marimuthu Palaniswami. "Influence of psychological stress on QT interval." In Computing in Cardiology 2014, pp. 1009-1012. IEEE, 2014

[28] Paavonen, K. J., Heikki Swan, Kirsi Piippo, Laura Hokkanen, Päivi Laitinen, Matti Viitasalo, Lauri Toivonen, and Kimmo Kontula. "Response of the QT interval to mental and physical stress in types LQT1 and LQT2 of the long QT syndrome." Heart 86, no. 1 (2001): 39-44.

[29] Cacioppo, John T., Louis G. Tassinary, and Gary Berntson, eds. Handbook of psychophysiology. Cambridge university press, 2007

[30] Kreibig, Sylvia D. "Autonomic nervous system activity in emotion: A review." Biological psychology 84, no. 3 (2010): 394-421.

[31] Munla, Nermine, Mohamad Khalil, Ahmad Shahin, and Azzam Mourad. "Driver stress level detection using HRV analysis." In 2015 international conference on advances in biomedical engineering (ICABME), pp. 61-64. IEEE, 2015.

[32] Yu, Ya Jun, Zhan Yang, Beom-Seok Oh, Yong Kiang Yeo, Qinglai Liu, Guang-Bin Huang and Zhiping Lin. "Investigation on driver stress utilizing ECG signals with on-board navigation systems in use." In 2016 14th ECG signals with on-board navigation systems in use." In 2016 14th (ICARCV), pp. 1-6. IEEE, 2016.

[33] Subhani, Ahmad Rauf, Wajid Mumtaz, Mohamed Naufal Bin Mohamed Saad, Nidal Kamel, and Aamir Saeed Malik. "Machine learning framework for the detection of mental stress at multiple levels." IEEE Access 5 (2017): $13545-13556$.

[34] Rizwan, Md Fahim, Rayed Farhad, Farhan Mashuk, Fakhrul Islam, and Mohammad Hasan Imam. "Design of a biosignal based stress detection system using machine learning techniques." In 2019 International Conference on Robotics, Electrical and Signal Processing Techniques (ICREST), pp. 364 on Robotics, Electr.

[35] Wang, Cuiyan, Riyu Pan, Xiaoyang Wan, Yilin Tan, Linkang Xu, Cyrus $\mathrm{S}$. Ho, and Roger C. Ho. "Immediate psychological responses and associated factors during the initial stage of the 2019 coronavirus disease (COVID-19) epidemic among the general population in China." International journal of environmental research and public health 17, no. 5 (2020): 1729.

[36] Wang, Yenan, Yu Di, Junjie Ye, and Wenbin Wei. "Study on the public psychological states and its related factors during the outbreak of coronavirus disease 2019 (COVID-19) in some regions of China." Psychology, Health \& Medicine (2020): 1-10.

[37] S.M. Didar-Ul Islam, Md. Bodrud-Doza, Rafid Mahmud Khan, Md Abidul Haque, Mohammed A. Mamun, "Exploring COVID-19 stress and its factors in Bangladesh: A perception-based study", Heliyon,Volume 6, Issue 7,2020, e04399,ISSN

8440,https://doi.org/10.1016/j.heliyon.2020.e04399.

[38] The Math Works, Inc. MATLAB, version 2016a. Natick, MA: The Math Works, Inc., 2020. Accessed January1, 2020. https://www.mathworks.com/
Science in Electrical and Electronics Engineering (M.Sc.) from the same university. His research interest includes Biomedical Signal Processing, Fiber Optical Communication and VLSI Circuits.

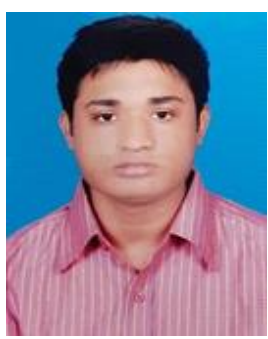

Rayed Farhad was born in Dhaka, Bangladesh. He obtained his Bachelor of Science degree in Electrical and Electronic Engineering from American International University - Bangladesh (AIUB), Dhaka, Bangladesh, in the year 2018. He started studying for postgraduate program in 2019, and currently pursuing degree for Masters of Science in Electrical and Electronics Engineering (M.Sc) from the same university. His research interest includes Biomedical Signal Processing, Fiber Optical Communication and Programming.

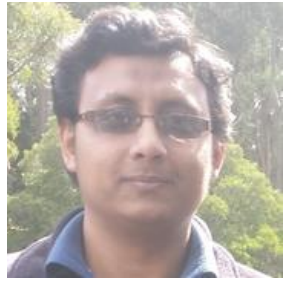

Mohammad Hasan Imam (MIEEE) Completed his B.sc Engg. from Bangladesh University of Engineering and Technology (BUET) in 2004 and Completed his $\mathrm{PhD}$ in 2015 from the University of Melbourne, Australia in the area of signal Processing especially Bio signal Processing and Modeling. He is currently working as Senior Assistant Professor in the department of Electrical and Electronic Engineering at the American International University-Bangladesh (AIUB). His main research interests include different topics in the area of bioengineering like Bioinformation processing, Model base system dynamics analysis, Machine learning in healthcare, Biomedical signal processing and Biomedical electronics.

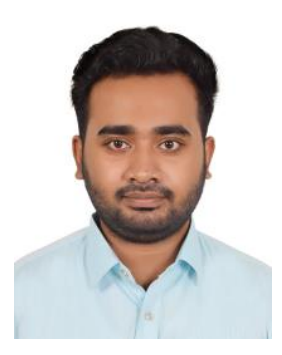

Md Fahim Rizwan was born in Thakurgaon, Bangladesh. He obtained his Bachelor of Science degree in Electrical and Electronic Engineering from American International University Bangladesh (AIUB), Dhaka, Bangladesh, in the year 2018. He started studying for post graduate program in 2019, and currently pursuing degree for Masters of 